\title{
NCCN Frameworks for Resource Stratification of NCCN Guidelines: Adult Cancer Pain and Palliative Care
}

Presented by Robert A. Swarm, MD, and Maria Dans, MD

\section{Abstract}

The NCCN Framework aims to provide adapted guidelines for low- and middle-resource countries to improve the experience of patients with cancer. In particular, the NCCN Frameworks for Adult Cancer Pain and Palliative Care and were designed to help expand access to pain management and palliative care for patients in low-resource countries. The NCCN Framework is one of several tools that can improve cancer care in the developing world. The NCCN Harmonized Guidelines for Sub-Saharan Africa, a collaborative effort between NCCN, American Cancer Society, Clinton Health Access Initiative, and African Cancer Coalition, was developed to harmonize NCCN recommendations with local guidelines across Africa and to make best use of available services and resources.

J Natl Compr Canc Netw 2018;16(5.5):628-631 doi: 10.6004/jnccn.2018.0044

More than 1 million people from 180 countries are registered users of the NCCN Guidelines, and almost half of these are global users. The NCCN Frameworks for Resource Stratification of NCCN Guidelines (NCCN Frameworks) for Adult Cancer Pain and Palliative Care are designed to help expand patient access to pain management and palliative care globally and to improve care in low-resource settings.

NCCN Guidelines document evidence-based, consensus-driven management to ensure that all patients receive optimal preventive, diagnostic services, and treatments that improve outcomes, explained Robert A. Swarm, MD, Professor of Anesthesiology, Washington University, and Chair of the NCCN Guidelines for Adult Cancer Pain, but not all countries have equal ac-

Presented by Robert A. Swarm, MD, Department of Anesthesiology, Siteman Cancer Center at Barnes-Jewish Hospital and Washington University, St. Louis, Missouri, and Maria Dans, MD, Siteman Cancer Center, Washington University, St. Louis, Missouri.

Drs. Swarm and Dans have disclosed that they have no financial interests, arrangements, affiliations, or commercial interests with the manufacturers of any products discussed in this article or their competitors.

Correspondence: Robert A. Swarm, MD, Department of Anesthesiology, Siteman Cancer Center at Barnes-Jewish Hospital and Washington University, Campus Box 8054, 660 South Euclid Avenue, St. Louis, MO 63110. Email: swarmr@wustl.edu; and

Maria Dans, MD, Siteman Cancer Center, Washington University, MailStop 90-29-931, 4590 Children's Place, St. Louis, MO 63110-1093.

Email: mariadans@wustl.edu cess to resources, and even within a high-resource utilization country like the United States there are pockets of low-resource utilization.

The United States has the highest costs associated with resource use, exceeding $\$ 10,000$ per patient per year. The United States also has high opioid consumption, which has increased over the past 20 years. "But vast numbers of patients with cancer throughout the world have little access to opioids," Dr. Swarm told the audience at the NCCN 23rd Annual Conference.

In October 2017, the Lancet Commission on Global Access to Palliative Care and Pain Relief showed that $90 \%$ of morphine is distributed to $10 \%$ of the world, and $1 \%$ was distributed to $50 \%$ of the world's population living in the poorest countries ( 3.6 billion people). "The uneven distribution of morphine has not gone unnoticed," Dr. Swarm stated.

The American Cancer Society's Treat the Pain program documents the number of treated deaths in pain and the number of untreated deaths in pain. ${ }^{2}$ There are large areas of the world where opioids are unavailable. For example, if India devoted $100 \%$ of the available opioids to the end of life, they still would not have enough resources to treat pain.

Worldwide, at least 2.3 million people died of HIV or cancer in untreated pain in 2013. ${ }^{2}$ In 2016, more than 63,000 drug-related deaths occurred in the United 
States, ${ }^{3}$ which is more than the number of US soldiers killed in Vietnam between 1955 and 1975, he said.

In low-resource countries such as India, morphine is primarily available for palliative care but is used by few doctors. Bureaucratic hurdles are significant, including depletion of supplies and frequent breaks in supplies. There are social, cultural, and infrastructural problems, as well as staffing shortages in low-resource countries. "Symptom control is not a focus of care," Dr. Swarm stated.

Even though access to opioids is limited in lowresource countries, it should be increased cautiously, with close marketing of supplies and strict conflict of interest policies in place for all phases of distribution and protocol development. Implementation of evidence-based treatment protocols is essential.

"Avoid overreliance on opioids. Use the NCCN Guidelines for Adult Cancer Pain," he noted. "Access to opioids is a surrogate for availability of cancer pain management, but there is a lot more to cancer pain management than access to opioids. Other strategies include psychological counseling, stress management, and integrative interventions," Dr. Swarm continued.

Global translation of the NCCN Guidelines entails evidence-based adaptations developed with local professional organizations and reviewed by international experts working regularly in low- and middle-resource clinical settings.

Resource-integrated adaptations of the NCCN Guidelines reflect the fluidity of resource availability, the ability to pay, rationing, uneven resource distribution, and unstable supply. The NCCN Framework attempts to address that need using a rational approach for constructing cancer management systems to provide the highest achievable level of care for every patient.

The NCCN Frameworks use 3 text colors to signify treatment recommendations applicable to each level of resource availability, Dr. Swarm explained. Black text signifies "include at that level"; gray text means "withhold at that level"; and italicized blue text means "a modified recommendation for that level." To demonstrate this, Dr. Swarm showed a page of the NCCN Guidelines for Adult Cancer Pain (Figure 1).

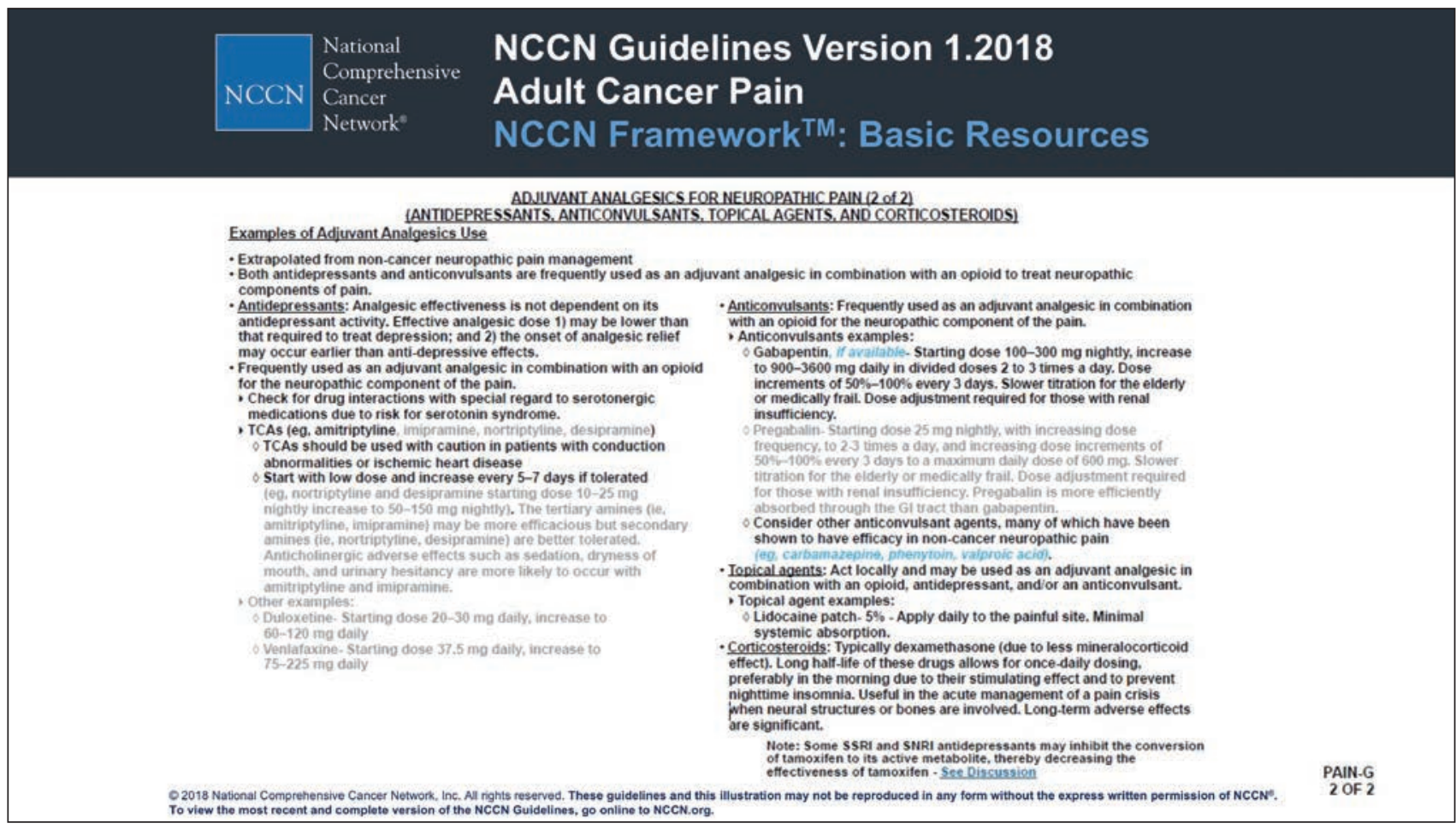

Figure 1. Algorithm page from the NCCN Guidelines for Adult Cancer Pain illustrating the NCCN Framework. Black text signifies "include at level"; grey text means "withhold at level"; and italicized blue text means "a modified recommendation for that level." 
"Today, $80 \%$ of all cancers are covered by the NCCN Framework," Dr. Swarm said.

\section{Harmonize Guidelines in Local Settings}

"NCCN Frameworks are only part of what's provided. Part of the process is to have the frameworks harmonize with local guidelines," said the next speaker, Maria Dans, MD, Assistant Professor of Medicine, Siteman Cancer Center, and Chair of the NCCN Palliative Care Panel. She noted that "NCCN is committed to expanding access to quality cancer care, including cancer pain management and palliative care, for all patients with cancer from the time of diagnosis until the time of death."

Dr. Dans explained that cancer is the second leading cause of mortality worldwide, with a total annual cancer cost in 2010 of $\$ 1.16$ trillion in the United States; $70 \%$ of cancer deaths occur in lowand middle-income countries (LMCs). Approximately $25 \%$ of deaths are related to cancer-causing infections, such as herpes virus, hepatitis C, and HIV. In LMCs, late-stage presentation and inaccessible diagnosis and treatment are common. Only 26\% of LMCs have available pathology service, and fewer than one-third have treatment services available in the public sector.

Other barriers to palliative care in LMCs include a focus on cure and lack of interventions that address pain and suffering but do not extend life. In addition to insufficient resources and lack of education about opioids, fear of opioid misuse and diversion leads to unbalanced and excessive laws and restrictions.

"The US epidemic of nonmedical opioid use is not seen in other high-income countries," Dr. Dans noted.

\section{How to Improve Access to Palliative Care and Pain Management?}

Dr. Dans suggested that the global AIDS response may suggest routes for increasing access to palliative care and pain management for patients with cancer in LMCs. "Advocacy can't provide all the answers," she said. "Advocacy tends to be disease-specific and focuses on cure and prevention."

She suggested that the NCCN Frameworks are an important tool, along with the WHO Model Lists

\section{Lancet Commission "Essential Package"}

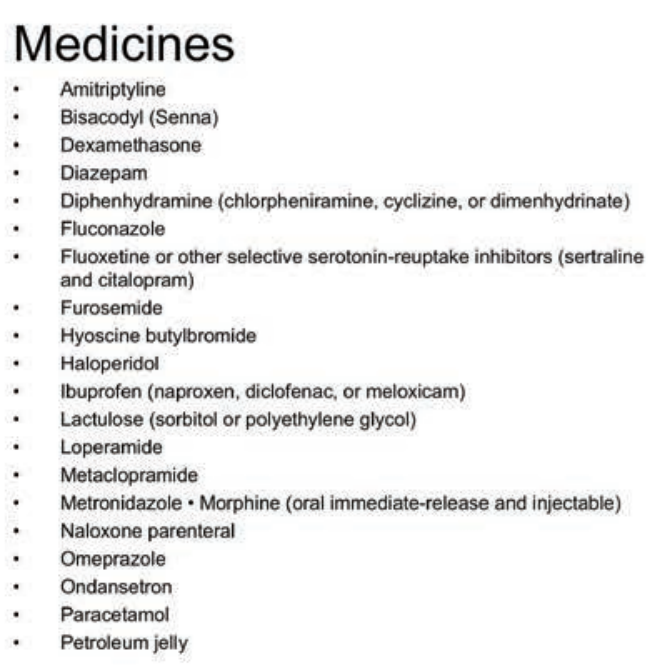

Medical equipment

- Pressure-reducing mattress

- Nasogastric drainage or feeding tube

- Urinary catheters

- Opioid lock box

- Flashlight with rechargeable battery (if no access to electricity)

- Adult diapers (or cotton and plastic, if in extreme poverty) - Oxygen

\section{Human resources (varies by} location \& venue of care)

- Doctors (specialty and general, depending on level of care)

- Nurses (specialty and general)

- Social workers and counsellors

- Psychiatrist, psychologist, or counsellor (depending on level of care)

- Physical therapist

- Pharmacist

- Community health workers

- Clinical support staff (diagnostic imaging, laboratory technician, nutritionist)

- Non-clinical support staff (administration, cleaning) 
of Essential Medicines ${ }^{4}$ and the Lancet Commission's "Essential Package of Palliative Care and Pain Relief Health Services"1 (Figure 2).

Dr. Dans then described her work on the NCCN Harmonized Guidelines for Sub-Saharan Africa. The goal of which was to harmonize cancer treatment guidelines across Africa and optimize resources for the best possible outcomes. These guidelines are evidence-based and updated annually, she noted.

The Harmonized Guidelines sought to highlight areas with available specialty and subspecialty care and identify areas where the parent NCCN Guidelines require modification.

"Cancer deaths in Sub-Saharan Africa are projected to reach 1 million by the year 2030, which is one-eighth of cancer deaths worldwide. Most cancer diagnoses are late-stage, and there is a shortage of pathology and treatment options," she explained.
In April and August of 2017, members of the African Cancer Coalition and NCCN met in Africa to review and adapt NCCN Guidelines. Eight NCCN Harmonized Guidelines for Sub-Saharan Africa were presented in Kigali, Rwanda, in November 2017 at the AORTIC meeting, including those for breast, cervical, and prostate cancers, B-cell lymphoma, chronic lymphocytic leukemia/small lymphocytic lymphoma, Kaposi sarcoma, adult cancer pain, and palliative care. Eight more guidelines will be released in 2018.

The NCCN Harmonized Guidelines also use different color texts to distinguish between levels of recommendations: black represents generally available standard of care; grey represents highly advanced technical care that may be costly and/or have a lesser impact on oncologic outcome. Blue text suggests regional options that may be considered when availability precludes standard of care.

\section{References}

1. Knaul FM, Farmer PE, Krakauer EL, et al. Alleviating the access abyss in palliative care relief - an imperative of universal health coverage: the Lancet Commission report. Lancet 2018;391:1391-1454.

2. American Cancer Society. Access to essential pain medicines brief (2013 data). Available at: http://treatthepain.org/Assets/ACCESS\%20TO\%20 ESSENTIAL\%20PAIN\%20MEDICINES\%20BRIEF.pdf. Accessed April 4, 2018.
3. Hedegaard H, Warner M, Minino AM. Drug overdose deaths in the United States, 1999-2016. National Center for Health Statistics, Centers for Disease control and Prevention. Available at: www.cdc.gov/nchs/products/ databriefs/db294.html. Accessed April 3, 2018.

4. WHO Model Lists of Essential Medicines. World Health Organization Web site. Available at: (http://www.who.int/medicines/publications/ essentialmedicines/en/. Accessed April 3, 2018. 